\title{
НЕПОСРЕДСТВЕННЫЕ РЕЗУЛЬТАТЫ ЛАПАРОСКОПИЧЕСКИХ РЕЗЕКЦИЙ ЖЕЛУДКА И ГАСТРЭКТОМИЙ ПРИ РАКЕ
}

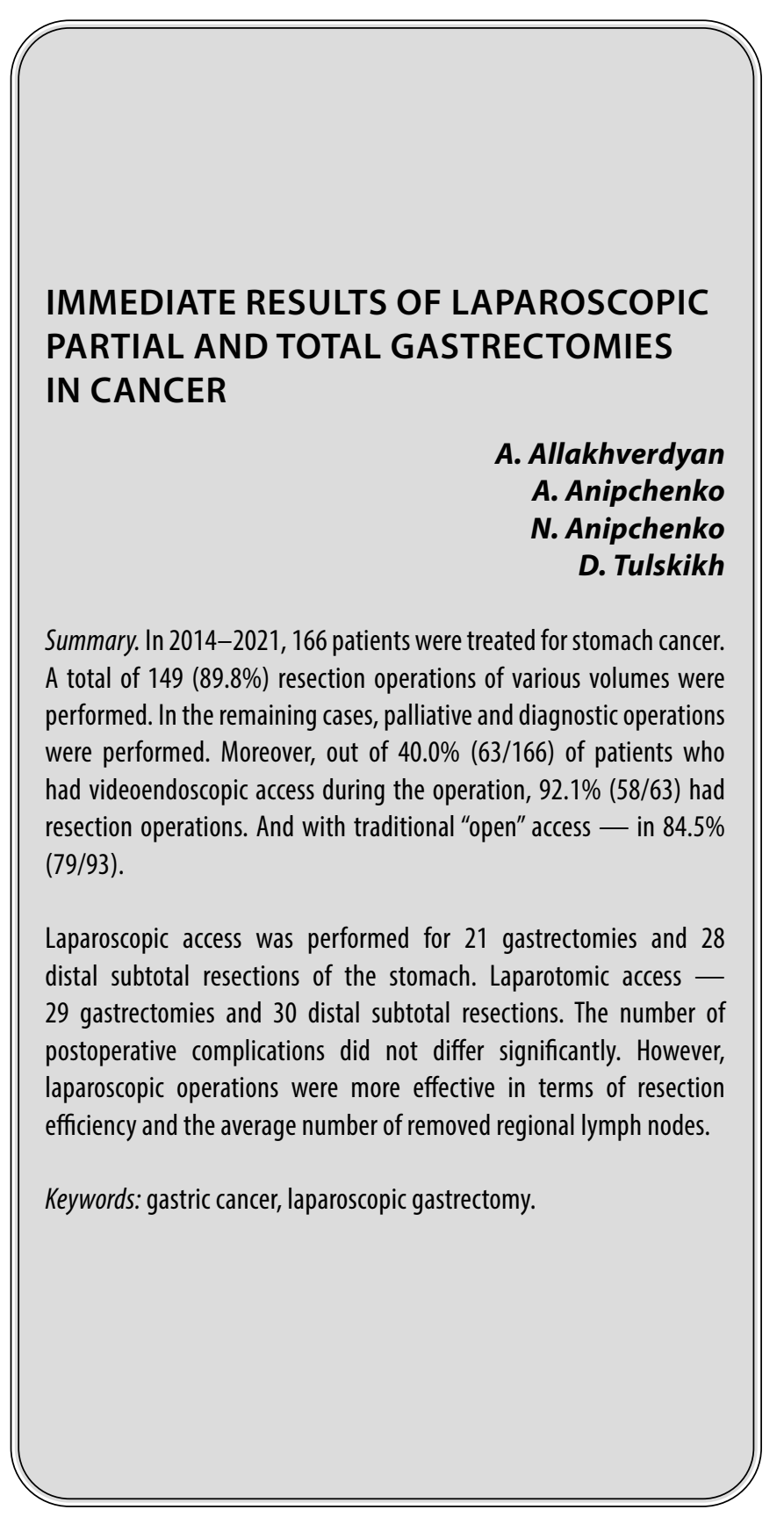

$\mathbf{P}$ езекция желудка или гастрэктомия остаются предпочтительным вариантом лечения рака желудка на ранних стадиях. Впервые радикальную лапароскопическую операцию (дистальную резекцию желудка) при раке в 1994 г. выполнил S. Kitano [1]. Преимущества подобных лапароскопических операций (низкое число послеоперационных осложнений и низ-
Аллахвердян Александр Сергеевич Д.м.н., профессор, ГБУЗ МО МОНИКИ им. М.Ф. Владимирского allakhverdyan@rambler.ru

Анипченко Алексей Николаевич К.м.Н., ГБУз МО МОНИКИ им. М.Ф. Владимирского dottor.alex@mail.ru

Анипченко Наталья Николаевна К.м.н., дочент, ГБУЗ МО МОНИКИ им. М.Ф. Владимирского dottor.na@gmail.com

Тульских Денис Александрович Ординатор, ГБУЗ МО МОНИКИ им. М.Ф. Владимирского denistulskih@yandex.ru

Аннотация. В 2014-2021 гг. по поводу рака желудка находились на лечении 166 пациентов. Всего было выполнено 149 (89,8\%) резекционных операций различных объемов. В остальных случаях были выполнены паллиативные и диагностические операции. Причем, из 40,0\% (63/166) пациентов у которых при операции были использованы видеоэндоскопические доступы, в 92,1\% (58/63) были выполнены резекционные операции. А при традиционных «открытых» доступах — в 84,5\% (79/93).

Лапароскопическим доступом была выполнена 21 гастрэктомия и 28 дистальных субтотальных резекций желудка. Лапаротомным доступом 29 гастрэктомий и 30 дистальных субтотальных резекций. Количество послеоперационных осложнений существенно не отличалось. Однако, лапароскопические операции были более эффективны по эффективности резекций и среднему количеству удаленных региональных лимфатических узлов.

Ключевые слова: рак желудка, лапароскопические гастрэктомии, лапароскопические резекции желудка.

кая летальность) продемонстрированы многими авторами $[2,3]$. При этом отмечено значительное уменьшение операционной травмы, сокращение уровня интраоперационной кровопотери, уменьшение уровня послеоперационного болевого синдрома и потребности в использовании анальгетиков. И у данной категории пациентов происходит быстрое восстановление 
Таблица 1. Количество и виды хирургических операций

\begin{tabular}{|c|c|c|}
\hline Доступ & Объем операции & $\begin{array}{l}\text { Количество } \\
\text { пациентов }\end{array}$ \\
\hline \multicolumn{3}{|l|}{ Миниинвазивные видеоэндоскопические доступы } \\
\hline \multirow{4}{*}{ Лапароскопический } & Гастрэктомия & 20 \\
\hline & Дистальная резекция желудка & 28 \\
\hline & Гастро-энтеростомия по Брауну & 2 \\
\hline & Гастростомия & 3 \\
\hline \multirow[t]{2}{*}{ Лапароскопический трансмедиастинальный } & \begin{tabular}{|l} 
Проксимальная \\
резекция желудка и нижнегрудного отдела пищевода
\end{tabular} & 8 \\
\hline & Гастрэктомия с резекцией нижней трети пищевода & 1 \\
\hline $\begin{array}{l}\text { Комбинированный Лапароскопия+Торакоскопия } \\
\text { справа (типа Льюиса) }\end{array}$ & $\begin{array}{l}\text { Проксимальная } \\
\text { резекция желудка и нижнегрудного отдела пищевода }\end{array}$ & 1 \\
\hline Всего & & 63 \\
\hline \multicolumn{3}{|l|}{ Традиционные «открытые» доступы } \\
\hline \multirow{6}{*}{ Лапаротомный } & Гастрэктомия & 29 \\
\hline & Проксимальная резекция желудка & 6 \\
\hline & Дистальная резекция желудка & 30 \\
\hline & Обходной гастроэнтеро-анастомоз по Брауну & 9 \\
\hline & Гастростомия & 3 \\
\hline & Диагностическая & 2 \\
\hline \multirow{2}{*}{ Абдоминоторакальный слева по Гэрлоку } & $\begin{array}{l}\text { Проксимальная резекция желудка с резекцией н/3 } \\
\text { пищевода }\end{array}$ & 6 \\
\hline & Гастрэктомия с резекцией н/3 пищевода & 18 \\
\hline \multicolumn{2}{|l|}{ Всего } & 93 \\
\hline \multicolumn{2}{|l|}{ ИТОГО } & 166 \\
\hline
\end{tabular}

и выявляется меньшее количество послеоперационных осложнений, чем при традиционном лапаротомном доступе [3, 4].

Уже есть доказательства того, что лапароскопическая дистальная резекция желудка является эффективной и с точки зрения онкологических результатов. Несмотря на техническую сложность выполнения лапароскопической гастрэктомии, методика не уступает по отдаленным результатам и общей выживаемости пациентов традиционной «открытой» операции [5].

Однако считается, что ограничения, возникающие при выполнении лапароскопической гастрэктомии, такие как относительно узкое поле зрения, невозможность непосредственной пальпации органов и тканей, сложности при манипуляциях во время выполнения лимфодиссекции D2, могут быть преодолены только при осуществлении операции опытными хирургами, что может влиять на результаты $[6,7]$.

С другой стороны, несмотря на понятные преимущества лапароскопической хирургии, имеется ограниченное количество работ, в которых были бы представлены доказательства полноценности лапароскопических операций, особенно в отношении местнораспространенного рака желудка, который требует обширной лимфодиссекции [8, 9, 10] Также нет исследований, в которых бы проводилась объективная оценка травматичности операций, основанная на принципах доказательной медицины.

Очевидна необходимость разработки не только оптимальной техники лапароскопических операций (гастрэктомии и дистальной субтотальной резекции) при местнораспространенном раке желудка, включая этап лимфодиссекции, но, и проведения объективной оценки уровня травматичности и онкологической полноценности подобных операций.

\section{Шель работы}

Изучение непосредственных результатов лапароскопической дистальной субтотальной резекции желудка и гастрэктомии при местнораспространенном раке желудка.

\section{Материалы и метолы}

В хирургическом торакальном отделении и онкологическом отделении хирургических методов лечения Московского областного научно-исследовательского клинического института им. М.Ф. Владимирского в 2014-2021 гг. находились на лечении 166 пациентов 
по поводу рака желудка. Средний возраст больных составил 61,9 лет. Среди них было 105 мужчин и 61 женщина. Всего было выполнено $149(89,8 \%)$ резекционных операций различных объемов (таблица 1.). В остальных случаях были выполнены паллиативные и диагностические операции. Причем, из 40,0\% (63/166) пациентов у которых при операции были использованы видеоэндоскопические доступы, в 92,1\% (58/63) были выполнены резекционные операции. А при традиционных «открытых» доступах — в 84,5\% (79/93).

Лапароскопическим доступом была выполнена 21 гастрэктомия. При этом в одном случае была выполнена трансмедиастинальная резекция нижнегрудного отдела пищевода с аппаратным пищеводно-кишечным анастомозом на уровне ретроперикардиального отдела пищевода. Традиционными «открытыми» доступами было выполнено 47 гастрэктомий: 29 гастрэктомий лапаротомным доступом; 18 гастрэктомий с резекцией нижнегрудгного отдела пищевода чресплевральным доступом по Гэрлоку.

Было выполнено 58 дистальных субтотальных резекций желудка: 30 операций лапаротомным доступом; 28 операций лапароскопическим доступом.

Всего была выполнена 21 проксимальная резекция желудка: 8 операций было выполнено с резекцией нижнегрудного отдела пищевода лапароскопическим трансмедиастинальным доступом; 1 проксимальная резекция желудка и нижнегрудного отдела пищевода была выполнена лапароскопическим комбинированным с торакоскопическим доступом (типа Льюиса); 6 резекций проксимального отдела желудка были выполнены лапаротомным доступом; 6 резекций проксимального отдела желудка и нижнегрудного отдела пищевода абдоминоторакальным доступом слева по Гэрлоку.

\section{Результаты}

При выполненных лапароскопическим доступом дистальных субтотальных резекции R0 были выполнены 28 (100\%) больным. При выполненных лапаротомных доступом дистальных субтотальных резекциях резекции R0 были выполнены 29 (96,6\%), R1-1 (3,3\%) из 30 больных. Разница статистически не достоверна. Во всех случаях выполнялась лимфодиссекция D2. После операций, выполненных торакоскопическом доступом, при гистологическом исследовании в среднем иссле-

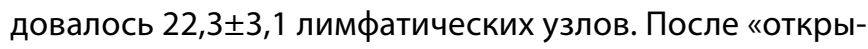
тых» - в среднем исследовалось 20,7士4,1 лимфатических узла. Разница достоверна.

При выполненных лапароскопическим доступом гастрэктомиях резекции R0 были выполнены всем 21
(100\%) больным. При выполненных лапаротомным доступом гастрэктомиях резекции R0 были выполнены 29 (100\%).

Мобилизация больных после лапароскопических операций происходила в первые сутки после операции. После операций, выполненных лапаротомным доступом, - на 2-3 сутки. Кормление per os начинали после ренгеновского контрастного исследования анастомозов на 5-6 сутки во всех группах пациентов.

После лапароскопических операций осложнения были отмечены всего у 4 из 49 больных (8,2\%). В 2-х случаях это были локальные несостоятельности швов пищеводно-кишечного анастомоза, излеченные консервативно. В одном случае послеоперационная пневмония и токсический гепатит у четвертого пациента. Средняя продолжительность нахождения в реанимации составила 17 ч. Средний послеоперационный койко-день при отсутствии осложнений составил 8 сут.

После лапаротомных операций осложнения были отмечены всего у 5 из 59 больных (8,5\%). В 2-х случаях это были локальные несостоятельности швов пищеводно-кишечного анастомоза, излеченные консервативно. В двух случаях послеоперационная пневмония. В одном случае был выявлен острый инфаркт миокарда. Средняя продолжительность нахождения в реанимации составила 19,5 ч. Средний послеоперационный койко-день при отсутствии осложнений составил 12 суток.

\section{Зак^ючение}

После лапаротомных операций количество осложнений достоверно не отличалось. Но отмечена существенная разница в пользу видеолапароскопии в сроках нахождения в отделении реанимации и интенсивной терапии и длительности послеоперационного периода. Также лапароскопические операции были более эффективны по эффективности резекций и среднему количеству удаленных региональных лимфатических узлов.

Видеоэндоскопические лапароскопические доступы не ограничивают возможности выполнения расширенных и комбинированных гастрэктомий и дистальных резекций при раке желудка.

Видеоэндоскопические лапароскопические доступы позволяют повысить эффективность хирургического лечения и обеспечивают четкость визуализации, хороший контроль гемостаза, удобство прецизионной мобилизации, раннюю активизацию больных в послеоперационном периоде, сокращение сроков стационарного лечения. 
ЛИТЕРАТУРА

1. Kitano S., Iso Y., Moriyama M., Sugimachi K. Laparoscopy-assisted Billroth I gastrectomy.// Surg Laparosc Endosc. 1994 Apr 4(2) — p.146-8.

2. Martin AN., Das D., Turrentine F.E., Bauer T.W., Adams R.B., Zaydfudim V.M. Morbidity and Mortality After Gastrectomy: Identification of Modifiable Risk Factors // J Gastrointest Surg- 2016 -Sep;20(9):1554-64.

3. Aratani K., Sakuramoto S., Chuman M. et al. Laparoscopy-assisted Distal Gastrectomy for Gastric Cancer in Elderly Patients: Surgical Outcomes and Prognosis.// Anticancer Res. - 2018 - Mar 38(3) - p. 1721-1725.

4. Liu F., Huang C., Xu Z. et al. Morbidity and Mortality of Laparoscopic vs Open Total Gastrectomy for Clinical Stage I Gastric Cancer: The CLASS02 Multicenter Randomized Clinical Trial.// Chinese Laparoscopic Gastrointestinal Surgery Study (CLASS) Group. — JAMA Oncol._ 2020 — 0ct 1; 6(10) — p. 1590 -1597.

5. Hyung W.J., Yang H.K., Park Y.K. et al. Long-Term Outcomes of Laparoscopic Distal Gastrectomy for Locally Advanced Gastric Cancer: The KLASS-02-RCT Randomized Clinical Trial. Korean Laparoendoscopic Gastrointestinal Surgery Study Group.// J Clin Oncol. — 2020 — 0ct 1;38(28) — p. $3304-3313$.

6. Özer İ., Bostancı E.B., Ulaş M., Özoğul Y., Akoğlu M. Changing Trends in Gastric Cancer Surgery.// Balkan Med J.— 2017 — Jan;34(1) — p.10-20.

7. Katai H., Mizusawa J., Katayama H. et. al. Single-arm confirmatory trial of laparoscopy-assisted total or proximal gastrectomy with nodal dissection for clinical stage I gastric cancer: Japan Clinical Oncology Group study JCOG1401. //Stomach Cancer Study Group of Japan Clinical Oncology Group.Gastric Cancer. — 2019 — Sep;22(5) - p.999-1008.

8. Yu J., Huang C., Sun Y. et al. Effect of Laparoscopic vs Open Distal Gastrectomy on 3-Year Disease-Free Survival in Patients With Locally Advanced Gastric Cancer: The CLASS-01 Randomized Clinical Trial. Chinese Laparoscopic Gastrointestinal Surgery Study (CLASS) Group.// JAMA. — 2019 — May 28; 321(20)- p. 19831992.

9. Garbarino G.M., Costa G., Laracca G.G. et al. Laparoscopic versus open distal gastrectomy for locally advanced gastric cancer in middle-low-volume centers in Western countries: a propensity score matching analysis. // Langenbecks Arch Surg. — 2020 — Sep;405(6) — p.797-807.

10. Schröder W, Bruns CJ. [Laparoscopic resection for locally advanced gastric cancer]. // Chirurg. — 2020 — Jan;91(1)- p.79-80.

( с Аллахвердян Александр Сергеевич ( allakhverdyan@rambler.ru), Анипченко Алексей Николаевич (dottor.alex@mail.ru ), Анипченко Наталья Николаевна ( dottor.na@gmail.com ), Тульских Денис Александрович ( denistulskih@yandex.ru ).

Журнал «Современная наука: актуальные проблемы теории и практики»

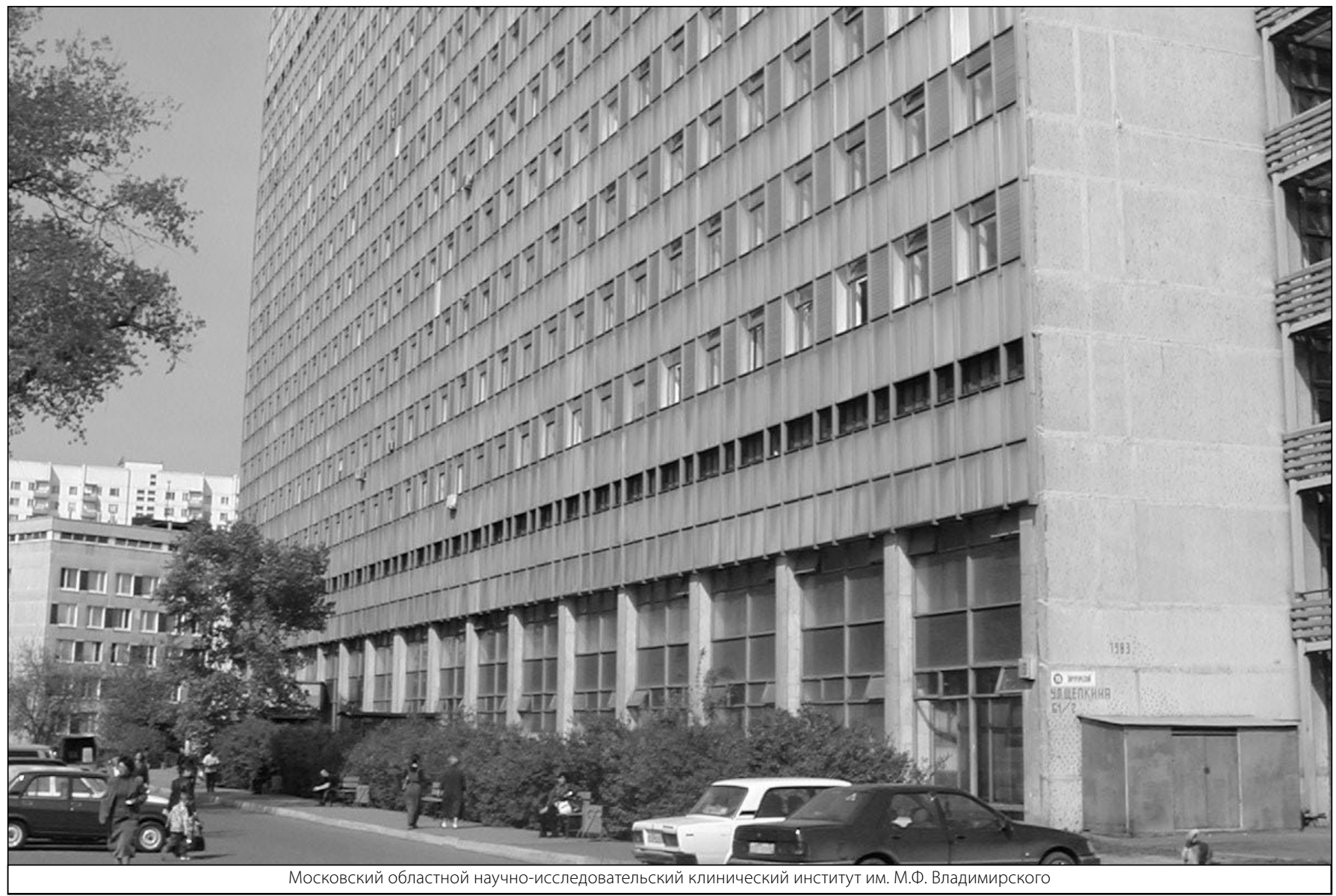

